CUBO A Mathematical Journal

Vol.21, No 01, (21-35). April 2019

\title{
Some New Simple Inequalities Involving Exponential, Trigonometric and Hyperbolic Functions
}

\author{
Yogesh J. Bagul ${ }^{1}$, Christophe Chesneau ${ }^{2}$ \\ ${ }^{1}$ Department of Mathematics, K. K. M. College Manwath, \\ Parbhani(M.S.) - 431505, India \\ ${ }^{2}$ LMNO, University of Caen Normandie, France \\ christophe. chesneau@unicaen.fr
}

\begin{abstract}
The prime goal of this paper is to establish sharp lower and upper bounds for useful functions such as the exponential functions, with a focus on $\exp \left(-x^{2}\right)$, the trigonometric functions (cosine and sine) and the hyperbolic functions (cosine and sine). The bounds obtained for hyperbolic cosine are very sharp. New proofs, refinements as well as new results are offered. Some graphical and numerical results illustrate the findings.
\end{abstract}

\section{RESUMEN}

El objetivo principal de este artículo es establecer cotas inferiores y superiores precisas para funciones útiles tales como las funciones exponenciales, con énfasis especial en $\exp \left(-\chi^{2}\right)$, las funciones trigonométricas (coseno y seno) y las funciones hiperbólicas (coseno y seno). Las cotas obtenidas para el coseno hiperbólico son muy precisas. Se presentan, tanto nuevas demostraciones y refinamientos, como resultados nuevos. Algunos resultados numéricos y gráficos ilustran los resultados encontrados.

Keywords and Phrases: Exponential function; trigonometric function; hyperbolic function. 2010 AMS Mathematics Subject Classification: 26D07, 33B10, 33B20. 


\section{Introduction}

Sharp bounds for useful functions play a central role in many areas of mathematics and theoretical physics. They aim to provide some properties of functions of interest, possibly complex, by dealing with more tractable functions (in the context). The literature on the bounds dealing with the special functions such as $e^{-x^{2}}, \cos (x), \sin (x), \operatorname{sinc}(x), \cosh (x), \sinh (x)$ and $\tanh (x)$, is very vast. Recent developments can be found in [10, 11, 7, 5, 1, 20, 17, 4, 15, 6, 21, 16, 3, 8, 14, 13, 18, 19, and the references therein. In this paper, we offer new simple tight (lower and upper) bounds involving these functions, with a high potential of interest for many researchers in mathematics or theoretical physics. Some proofs of our results are based on the so-called l'Hospital's rule of monotonicity, the others used recent results with a new approach. The sharpness of our bounds are highlighted by some graphics and numerical studies using a global $\mathrm{L}_{2}$ error as benchmark.

The result below shows bounds for $e^{-x^{2}}$ defined with the cosine function and well-chosen constants.

Proposition 1.1. For $\chi \in(0, \pi / 2)$, the best possible constants $\alpha$ and $\beta$ in the following inequalities

$$
\frac{\cos (x)-1+\alpha}{\alpha} \leqslant e^{-x^{2}} \leqslant \frac{\cos (x)-1+\beta}{\beta}
$$

are $1 / 2$ and $\approx 1.092663$ respectively.

The interest of Proposition 1.1 is the simplicity of the bounds, with very tractable expressions. It can be useful to evaluate complex functions depending on $e^{-x^{2}}$ (Gaussian probability density function, error function etc.). The bounds of Proposition 1.1 are illustrated in Figure 1. We see that the lower bound is sharp for small values for $x$.

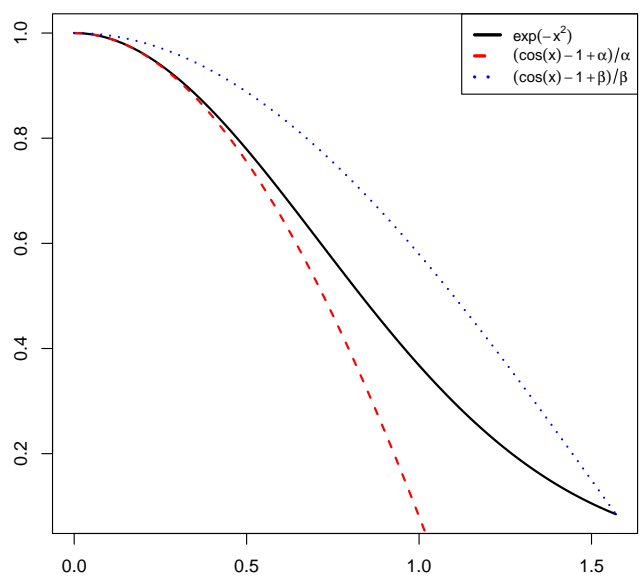


Figure 1: Graphs of the functions of the bounds (1.1) for $x \in(0, \pi / 2)$.

Note: Using exponential and cosine series, Proposition 1.1 can be expressed in terms of alternating series as follows.

For $x \in(-\pi / 2, \pi / 2)$, we have

$$
\frac{1}{\alpha} \sum_{k=1}^{\infty} \frac{(-1)^{k} \chi^{2 k}}{(2 k) !} \leqslant \sum_{k=1}^{\infty} \frac{(-1)^{k} \chi^{2 k}}{k !} \leqslant \frac{1}{\beta} \sum_{k=1}^{\infty} \frac{(-1)^{k} \chi^{2 k}}{(2 k) !},
$$

where $\alpha$ and $\beta$ are as defined above.

Now let us recall that the sinc function is defined by

$$
\operatorname{sinc}(x)=\left\{\begin{array}{cc}
\frac{\sin (x)}{x} & x \neq 0, \\
1 & x=0 .
\end{array}\right.
$$

It is of importance due to it's frequent occurrence in Fourier analysis. So the interest of finding the bounds of this type of functions is increasing. In the next proposition, we give new bounds to sinc function using hyperbolic tangent.

Proposition 1.2. For $x \in(0, \pi / 2)$, we have

$$
\left(\frac{\tanh (x)}{x}\right)^{\delta}<\frac{\sin (x)}{x}<\left(\frac{\tanh (x)}{x}\right)^{\eta}
$$

with the best possible constants $\delta=0.839273$ and $\eta=1 / 2$.

In the following propositions, the inequalities presented are somewhat Cusa-Huygen's type [13, 18. Proposition 1.3 below provides bounds for the sinc function using $e^{-x^{2}}$ or hyperbolic cosine.

Proposition 1.3. For $x \in(0, \pi / 2)$, the inequalities

$$
\left(\frac{2+e^{-x^{2}}}{3}\right)^{a}<\frac{\sin (x)}{x}<\left(\frac{2+e^{-x^{2}}}{3}\right)^{b}
$$

and

$$
\left(\frac{3}{2+\cosh (x)}\right)^{c}<\frac{\sin (x)}{x}<\left(\frac{3}{2+\cosh (x)}\right)^{d}
$$

are true with the best possible constants $\mathrm{a} \approx 1.240827, \mathrm{~b}=1 / 2, \mathrm{c} \approx 1.108171$ and $\mathrm{d}=1$. 
In view of Propositions 1.2 and 1.3 it is natural to address the following question: Which bounds for sinc are the best? We provide the answer by doing a numerical study. We investigate the global $\mathrm{L}_{2}$ error defined by

$$
e(u)=\int_{0}^{\pi / 2}\left(\frac{\sin x}{x}-u(x)\right)^{2} d x
$$

where $\mathfrak{u}(\mathrm{x})$ denotes bound (lower or upper) in (1.3), (1.4) and (1.5). The results are summarized in Table1.

Table 1: Global $L_{2}$ errors $e(u)$ for $\operatorname{sinc}(x)$ and the functions $u(x)$ in the bounds of (1.3), (1.4)

\begin{tabular}{|c|c|c|c|}
\hline \multirow{9}{*}{ and (1.5) for $x \in(0, \pi / 2)$. } & & \multicolumn{2}{|l|}{ Inequality (1.3) } \\
\hline & $u(x)$ & lower & upper \\
\hline & $e(u)$ & $\approx 0.001421437$ & $\approx 0.003648618$ \\
\hline & & \multicolumn{2}{|l|}{ Inequality (1.4) } \\
\hline & $u(x)$ & lower & upper \\
\hline & $e(u)$ & $\approx 0.006242974$ & $\approx 0.008628254$ \\
\hline & & \multicolumn{2}{|l|}{ Inequality (1.5) } \\
\hline & $u(x)$ & lower & upper \\
\hline & $e(u)$ & $\approx 6.53313 \times 10^{-5}$ & $\approx 0.0001542441$ \\
\hline
\end{tabular}

It follows from Table 1 that the bounds (1.5) are more sharp. This sharpness is illustrated in Figure 2.

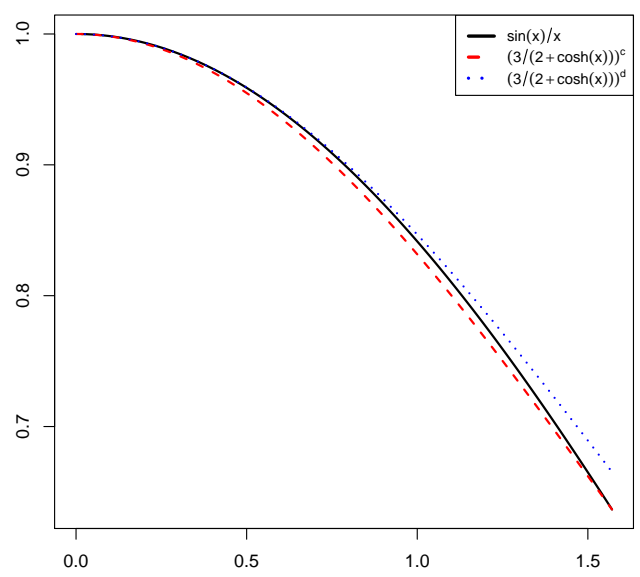

Figure 2: Graphs of the functions of the bounds (1.5) for $x \in(0, \pi / 2)$. 
The next result provides bounds for $x / \sinh (x)$ using cosine function.

Proposition 1.4. If $x \in(0, \pi / 2)$ then we have

$$
\left(\frac{2+\cos (x)}{3}\right)^{m}<\frac{x}{\sinh (x)}<\left(\frac{2+\cos (x)}{3}\right)^{n}
$$

with the constants $\mathrm{m} \approx 1.014227$ and $\mathrm{n} \approx 0.928648$.

The obtained bounds are illustrated in Figure 3.

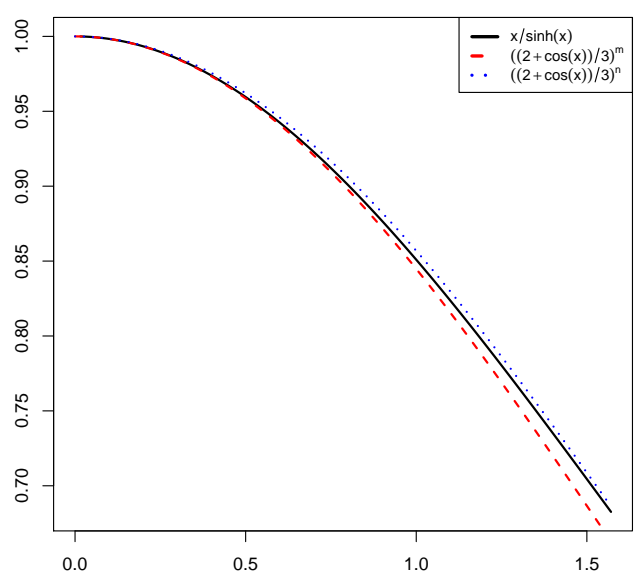

Figure 3: Graphs of the functions of the bounds (1.6) for $x \in(0, \pi / 2)$.

Note: The inequality

$$
\frac{2+\cos (x)}{3}<\frac{x}{\sinh (x)}
$$

is more sharp version of left inequality of (1.6). It is appeared in [19, Theorem 6].

Proposition 1.5 below presents sharp bounds for $\sinh (\chi) / \chi$ using hyperbolic cosine.

Proposition 1.5. For $x \in(0, \pi / 2)$ one has

$$
\left(\frac{2+\cosh (x)}{3}\right)^{p}<\frac{\sinh (x)}{x}<\left(\frac{2+\cosh (x)}{3}\right)^{q}
$$

with the constants $\mathrm{p} \approx 0.928648$ and $\mathrm{q} \approx 1.009155$.

The bounds are illustrated in Figure 4. 


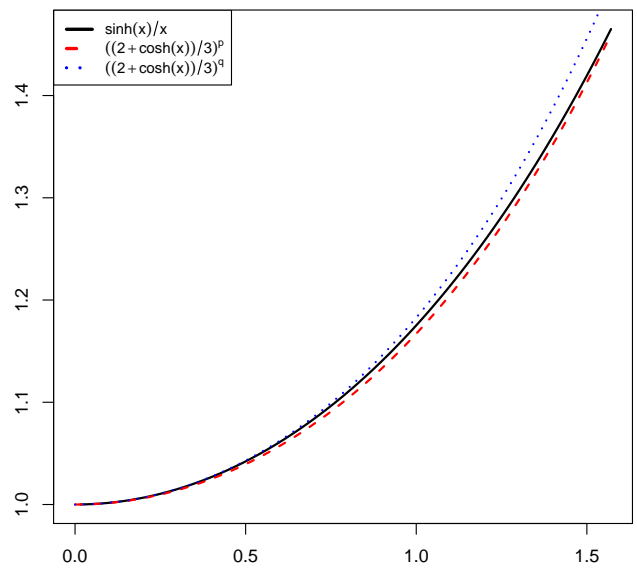

Figure 4: Graphs of the functions of the bounds (1.7) for $x \in(0, \pi / 2)$.

Note: The hyperbolic Cusa-Huygen's inequality 16

$$
\frac{\sinh (x)}{x}<\frac{2+\cosh (x)}{3}
$$

is however more sharp than right inequality of (1.7).

The rest of the study is devoted to new bounds for $\cosh (x)$, with discussion. A well-known upper bound for $\cosh (x)$ is given by $e^{x^{2} / 2}$. This result was recently completed by Yogesh Bagul[3, Theorem 2.1] who finds a sharp lower bound, i.e.

$$
\mathrm{e}^{\mathrm{ax} \mathrm{x}^{2}}<\cosh (\mathrm{x})<\mathrm{e}^{\mathrm{x}^{2} / 2}, \quad x \in(0,1),
$$

with the best possible constants $a \approx 0.433781$ and $1 / 2$. We now aim to refine the inequalities of (1.8) in Proposition 1.6 below.

Proposition 1.6. For $x \in(0,1)$, we have

$$
\exp \left(\frac{3}{2}\left(1-e^{-x^{2} / 3}\right)\right) \leqslant \cosh (x) \leqslant \exp \left(\frac{1}{2 \theta}\left(1-e^{-\theta x^{2}}\right)\right)
$$

with $\theta \approx 0.272342$.

Note: Using the well-known inequality $e^{y} \geqslant 1+y$ for $y \in \mathbb{R}$, we obtain $\exp \left(\left(1-e^{-\theta x^{2}}\right) /(2 \theta)\right) \leqslant$ $e^{x^{2} / 2}$. This proves that the upper bound in (1.9) is sharper to the one in (1.8).

Alternative bounds are given in Proposition 1.7 below, with discussion. 
Proposition 1.7. For $x \in(0,1)$, we have

$$
\left(1+\frac{x^{2}}{3}\right)^{3 / 2} \leqslant \cosh (x) \leqslant\left(1+\frac{x^{2}}{\xi}\right)^{\xi / 2}
$$

with $\xi \approx 3.194528$.

Note: Again, using the well-known inequality $e^{y} \geqslant 1+y$ for $y \in \mathbb{R}$, we get $\left(1+x^{2} / \xi\right)^{\xi / 2} \leqslant$ $e^{x^{2} / 2}$. This shows that the upper bound in (1.10) is sharper to the one in (1.8).

We now claim that the bounds obtained in (1.10) are better than those in (1.8) and (1.9). Numerical results support this claim. Indeed, by considering the global $L_{2}$ error defined by

$$
e_{*}(u)=\int_{0}^{1}(\cosh (x)-u(x))^{2} d x
$$

where $u(x)$ denotes bound (lower or upper) in (1.8), (1.9) and (1.10), Table 1 indicates that (1.10) are the best.

Table 2: Global $\mathrm{L}_{2}$ errors $e_{*}(u)$ for $\cosh (x)$ and the functions $u(x)$ in the bounds of (1.8), (1.9)

\begin{tabular}{|c|c|c|}
\hline & \multicolumn{3}{|l|}{ Inequality (1.8) } \\
\hline$u(x)$ & lower & upper \\
\hline$e_{*}(u)$ & $\approx 0.0001352084$ & $\approx 0.001139289$ \\
\hline \hline & Inequality (1.9) \\
\hline$u(x)$ & lower & upper \\
\hline$e_{*}(u)$ & $\approx 1.335929 \times 10^{-5}$ & $\approx 7.004029 \times 10^{-6}$ \\
\hline \hline & Inequality (1.10) \\
\hline$u(x)$ & lower & upper \\
\hline$e_{*}(u)$ & $\approx 9.456552 \times 10^{-7}$ & $\approx 6.895902 \times 10^{-7}$ \\
\hline
\end{tabular}

The sharpness of the obtained bounds is illustrated in Figures 5 and 6 (for a zoom on the interval $(0.95,1)$, where the hierarchy of the bounds is more clear). 


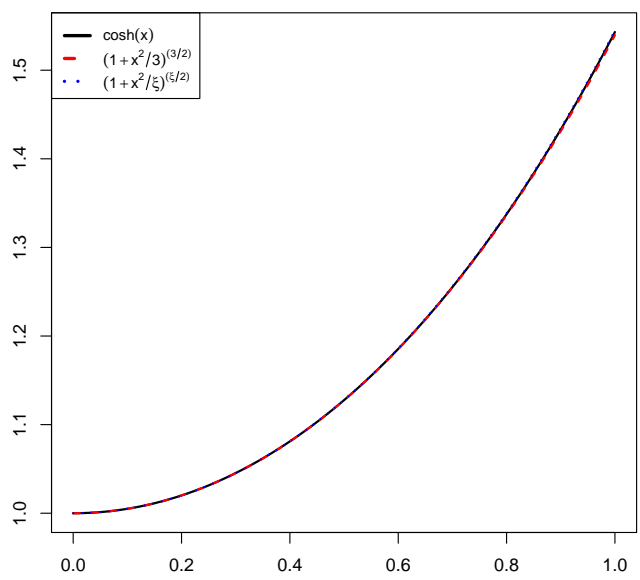

Figura 5: Graphs of the functions of the bounds (1.10) for $x \in(0,1)$.

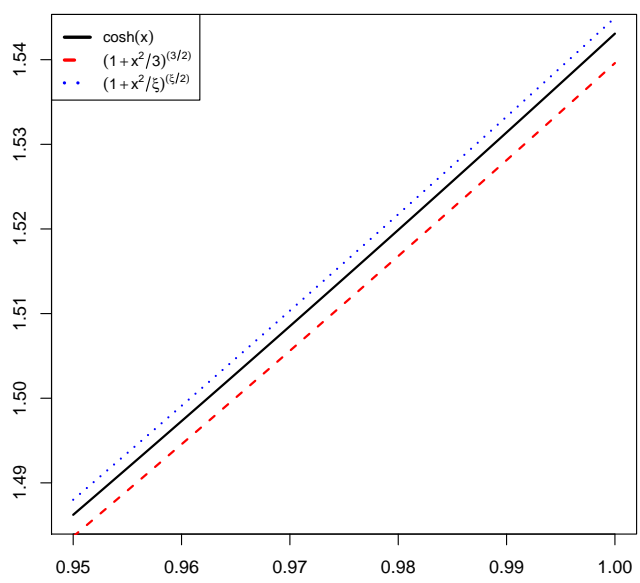

Figura 6: Graphs of the functions of the bounds (1.10) for $x \in(0.95,1)$.

Note: To prove the inequalities (1.5), (1.6) and (1.7), we will simply use the results of [7, 5, 12]. We stress on the fact that it is not difficult to verify that all the results in $[5$ are also true in $(0, \pi / 2)$ with the respective best possible constants obtained accordingly (see [12]). Propositions [1.6 and 1.7 will be proved by the techniques of integration on some known results [4, 6]. For proving Proposition 1.1. Proposition 1.2 and Proposition 1.3. we need the Lemmas presented in the next section. 


\section{Lemmas}

The following Lemma is known as l'Hospital's rule of monotonicity. The details are given in [9] and $[2]$.

Lemma 2.1. ([2]) Let $\mathrm{f}, \mathrm{g}$ be two real valued functions which are continuous on $[\mathrm{a}, \mathrm{b}]$ and differentiable on $(\mathrm{a}, \mathrm{b})$, where $-\infty<\mathrm{a}<\mathrm{b}<\infty$ and $\mathrm{g}^{\prime}(\mathrm{x}) \neq 0$, for $\forall x \in(\mathrm{a}, \mathrm{b})$. Let,

$$
A(x)=\frac{f(x)-f(a)}{g(x)-g(a)}
$$

and

$$
B(x)=\frac{f(x)-f(b)}{g(x)-g(b)}
$$

Then,

I) $\mathrm{A}(\mathrm{x})$ and $\mathrm{B}(\mathrm{x})$ are increasing on $(\mathrm{a}, \mathrm{b})$ if $\mathrm{f}^{\prime} / \mathrm{g}^{\prime}$ is increasing on $(\mathrm{a}, \mathrm{b})$ and II) $\mathrm{A}(\mathrm{x})$ and $\mathrm{B}(\mathrm{x})$ are decreasing on $(\mathrm{a}, \mathrm{b})$ if $\mathrm{f}^{\prime} / \mathrm{g}^{\prime}$ is decreasing on $(\mathrm{a}, \mathrm{b})$.

The strictness of the monotonicity of $\mathrm{A}(\mathrm{x})$ and $\mathrm{B}(\mathrm{x})$ depends on the strictness of monotonicity of $\mathrm{f}^{\prime} / \mathrm{g}^{\prime}$.

Lemma 2.2. $\mathrm{H}(\mathrm{x})=\frac{\sin (\mathrm{x})-\mathrm{x} \cos (\mathrm{x})}{\mathrm{x}^{2} \sin (\mathrm{x})}$ is strictly positive increasing in $(0, \pi / 2)$.

Proof: $H(x)$ is positive as $\cos (x)<\frac{\sin (x)}{x}$ on $(0, \pi / 2)$.

Consider,

$$
H(x)=\frac{\sin (x)-x \cos (x)}{x^{2} \sin (x)}=\frac{H_{1}(x)}{H_{2}(x)},
$$

where $H_{1}(x)=\sin (x)-x \cos (x)$ and $H_{2}(x)=x^{2} \sin (x)$ are such that $H_{1}(0)=0$ and $H_{2}(0)=0$. By differentiating

$$
\frac{H_{1}^{\prime}(x)}{H_{2}^{\prime}(x)}=\frac{\sin (x)}{x \cos (x)+2 \sin (x)}=\frac{H_{3}(x)}{H_{4}(x)},
$$

where $H_{3}(x)=\sin (x)$ and $H_{4}(x)=x \cos (x)+2 \sin (x)$ with $H_{3}(0)=0$ and $H_{4}(0)=0$. Again differentiating we get

$$
\frac{H_{3}^{\prime}(x)}{H_{4}^{\prime}(x)}=\frac{\cos (x)}{-x \sin (x)+3 \cos (x)}=\frac{1}{-x \tan (x)+3} .
$$

Now, it is well known that $-x \tan (x)$ is decreasing in $(0, \pi / 2)$ and so is $-x \tan (x)+3$. By Lemma $1, H(x)$ is a strictly increasing function in $(0, \pi / 2)$.

\section{Proofs of the Main Results}

This section is devoted to the proofs of our main results. 
Proof of Proposition 1.1; Clearly, the equalities hold at $x=0$. Consider

$$
f(x)=\frac{\cos (x)-1}{e^{-x^{2}}-1}=\frac{f_{1}(x)}{f_{2}(x)}
$$

where $f_{1}(x)=\cos (x)-1$ and $f_{2}(x)=e^{-x^{2}}-1$ with $f_{1}(0)=0$ and $f_{2}(0)=0$. By differentiation, we obtain

$$
\frac{f_{1}^{\prime}(x)}{f_{2}^{\prime}(x)}=\frac{\sin (x) e^{x^{2}}}{2 x}=\frac{f_{3}(x)}{f_{4}(x)}
$$

where $f_{3}(x)=\sin (x) e^{x^{2}}$ and $f_{4}(x)=2 x$ with $f_{3}(0)=0$ and $f_{4}(0)=0$. Again differentiating we get

$$
\begin{aligned}
\frac{f_{3}^{\prime}(x)}{f_{4}^{\prime}(x)} & =\frac{e^{x^{2}}}{2}[\cos (x)+2 x \sin (x)] \\
& =\frac{e^{x^{2}}}{2} F(x),
\end{aligned}
$$

where $F(x)=\cos (x)+2 x \sin (x)$. Differentiation gives

$$
\mathrm{F}^{\prime}(x)=2 x \cos (x)+\sin (x)>0
$$

in $(0, \pi / 2)$, which implies that $F(x)$ is increasing. Thus $\frac{f_{3}^{\prime}(x)}{f_{4}^{\prime}(x)}$ being a product of two positive increasing functions is a positive increasing. By Lemma 2.1, $f(x)$ is also increasing in $(0, \pi / 2)$. So $\alpha=f(0+)=1 / 2$ and $\beta=f(\pi / 2-)=-1 /\left[e^{-(\pi / 2)^{2}}-1\right] \approx 1.092663$.

Proof of Proposition 1.2, Let us set

$$
h(x)=\frac{\log (\sin (x) / x)}{\log (\tanh (x) / x)}=\frac{h_{1}(x)}{h_{2}(x)}
$$

where $h_{1}(x)=\log (\sin (x) / x)$ and $h_{2}(x)=\log (\tanh (x) / x)$ with $h_{1}(0+)=0$ and $h_{2}(0+)=0$. Differentiating we get

$$
\frac{h_{1}^{\prime}(x)}{h_{2}^{\prime}(x)}=\frac{\sin (x)-x \cos (x)}{x^{2} \sin (x)} \frac{x^{2} \tanh (x)}{\tanh (x)-x \operatorname{sech}^{2}(x)}=H(x) J(x)
$$

where $H(x)=\frac{\sin (x)-x \cos (x)}{x^{2} \sin (x)}$ and $J(x)=\frac{x^{2} \tanh (x)}{\tanh (x)-x \operatorname{sech}^{2}(x)}$. Now set

$$
J(x)=\frac{J_{1}(x)}{J_{2}(x)}
$$

where $J_{1}(x)=x^{2} \tanh (x)$ and $J_{2}(x)=\tanh (x)-x \operatorname{sech}^{2}(x)$ with $J_{1}(0)=0$ and $J_{2}(0)=0$. Differentiation gives

$$
\begin{aligned}
\frac{J_{1}^{\prime}(x)}{J_{2}^{\prime}(x)} & =\frac{x \operatorname{sech}^{2}(x)+2 \tanh (x)}{2 \operatorname{sech}^{2}(x) \tanh (x)} \\
& =\frac{1}{2} \frac{x}{\tanh (x)}+\cosh ^{2}(x),
\end{aligned}
$$


which is clearly increasing as both $x / \tanh (x)$ and $\cosh ^{2}(x)$ are increasing. By Lemma 2.1. $J(x)$ is also increasing in $(0, \pi / 2)$. Moreover, $J(x)$ is positive as $x / \sinh (x)<\cosh (x)$. By Lemma 2.2. $H(x)$ is strictly positive increasing in $(0, \pi / 2) \cdot h_{1}^{\prime}(x) / h_{2}^{\prime}(x)$, being product of two positive increasing functions is positive increasing. Again by Lemma 2.1, $h(x)$ is strictly increasing in $(0, \pi / 2)$. So $\delta=\log (2 / \pi) / \log (2 \tanh (\pi / 2) / \pi) \approx 0.839273$ and $\eta=f(0+)=1 / 2$, by l'Hospital's rule. This completes the assertion.

\section{Proof of Proposition 1.3;}

- Proof of (1.4). Let

$$
f(x)=\frac{\log (\sin (x) / x)}{\log \left(2+e^{-x^{2}}\right)-\log 3}=\frac{f_{1}(x)}{f_{2}(x)}
$$

where $f_{1}(x)=\log (\sin (x) / x)$ and $f_{2}(x)=\log \left(2+e^{-x^{2}}\right)-\log 3$ such that $f_{1}(0+)=0$ and $f_{2}(0)=0$. Differentiation gives

$$
\begin{aligned}
\frac{f_{1}^{\prime}(x)}{f_{2}^{\prime}(x)} & =\frac{1}{2} \frac{(\sin (x)-x \cos (x))}{x^{2} \sin (x)}\left(2 e^{x^{2}}+1\right) \\
& =\frac{1}{2} H(x) G(x),
\end{aligned}
$$

where $H(x)=\frac{\sin (x)-x \cos (x)}{x^{2} \sin (x)}$ is strictly positive increasing in $(0, \pi / 2)$ by Lemma 2.2 and $\mathrm{G}(\mathrm{x})=2 \mathrm{e}^{\mathrm{x}^{2}}+1$ is also clearly positive increasing. Therefore $\mathrm{H}(\mathrm{x}) \mathrm{G}(\mathrm{x})$ is strictly increasing. By making use of Lemma 2.1, we conclude that $f(x)$ is strictly increasing in $(0, \pi / 2)$. So

$$
f(0+)<f(x)<f(\pi / 2) ; x \in(0, \pi / 2) .
$$

Hence, $a=f(\pi / 2)=\log (2 / \pi) /\left[\log \left(2+e^{-(\pi / 2)^{2}}\right)-\log 3\right] \approx 1.240827$ and $b=f(0+)=1 / 2$ by l'Hospital's rule.

- Proof of (1.5). Utilizing [5, Theorem 2], 12, Proposition 3] we have

$$
e^{-k x^{2}}<\frac{\sin (x)}{x}<e^{-x^{2} / 6}
$$

where $k=\frac{-\log (2 / \pi)}{(\pi / 2)^{2}}$. After rearrangement, it can be written as

$$
\left(\frac{\sin (x)}{x}\right)^{6}<e^{-x^{2}}<\left(\frac{\sin (x)}{x}\right)^{1 / k} .
$$

By virtue of [7, Theorem 2] we write

$$
\left(\frac{3}{2+\cosh (x)}\right)^{\gamma}<e^{-x^{2}}<\left(\frac{3}{2+\cosh (x)}\right)^{6}
$$


where $\gamma=\frac{(\pi / 2)^{2}}{\log [(2+\cosh (\pi / 2)) / 3]}$. Combining (3.1) and (3.2), we get

$$
\left(\frac{3}{2+\cosh (x)}\right)^{c}<\frac{\sin (x)}{x}<\left(\frac{3}{2+\cosh (x)}\right)
$$

where $\mathrm{c}=\mathrm{k} \gamma=\frac{-\log (2 / \pi)}{\log [(2+\cosh (\pi / 2)) / 3]} \approx 1.108171$.

Proof of Proposition 1.4; According to [5, Theorem 3] and [12] we have

$$
e^{-x^{2} / 6}<\frac{x}{\sinh (x)}<e^{-t x^{2}}, x \in(0, \pi / 2)
$$

where $t=\frac{-\log [\pi /(2 \sinh (\pi / 2))]}{(\pi / 2)^{2}}$. It is equivalent to

$$
\left(\frac{x}{\sinh (x)}\right)^{1 / t}<e^{-x^{2}}<\left(\frac{x}{\sinh (x)}\right)^{6}
$$

Similarly, using [7, Theorem 1] we have

$$
\left(\frac{2+\cos (x)}{3}\right)^{\lambda}<e^{-x^{2}}<\left(\frac{2+\cos (x)}{3}\right)^{6},
$$

where $\lambda=\frac{-(\pi / 2)^{2}}{\log (2 / 3)}$. Combining (3.3) and (3.4) we get

$$
\left(\frac{2+\cos (x)}{3}\right)^{m}<\frac{x}{\sinh (x)}<\left(\frac{2+\cos (x)}{3}\right)^{n}
$$

where $\mathrm{m}=\frac{\lambda}{6}=\frac{-(\pi / 2)^{2}}{6 \log (2 / 3)} \approx 1.014227$ and $\mathrm{n}=6 \mathrm{t}=\frac{-6 \log [\pi /(2 \sinh (\pi / 2))]}{(\pi / 2)^{2}} \approx 0.928648$.

Proof of Proposition 1.5; The proof follows easily by combining inequalities (3.2) and (3.3) to get

$$
\mathrm{p}=\frac{-6 \log [\pi /(2 \sinh (\pi / 2))]}{(\pi / 2)^{2}} \approx 0.928648 \text { and } \mathrm{q}=\frac{(\pi / 2)^{2}}{6 \log [(2+\cosh (\pi / 2)) / 3]} \approx 1.009155
$$

Proof of Proposition 1.6; For $x=0$ equalities hold obviously. Rearranging [4, Theorem $5]$, for any $t \in(0,1)$, we have

$$
\mathrm{t} e^{-\mathrm{t}^{2} / 3}<\tanh (\mathrm{t})<\mathrm{t} e^{-\theta \mathrm{t}^{2}}
$$

with $\theta \approx 0.272342$. Therefore by integration, for $x \in(0,1)$, we get

$$
\int_{0}^{x} t e^{-t^{2} / 3} d t<\int_{0}^{x} \tanh (t) d t<\int_{0}^{x} t e^{-\theta t^{2}} d t
$$

which yields

$$
\frac{3}{2}\left(1-e^{-x^{2} / 3}\right)<\log (\cosh (x))<\frac{1}{2 \theta}\left(1-e^{-\theta x^{2}}\right) .
$$


By composing with the exponential function, we get the required result.

Proof of Proposition 1.7; Clearly, the equalities hold at $x=0$. Rearranging 6, Theorem $4]$, for any $t \in(0,1)$, we have

$$
\frac{3 \mathrm{t}}{3+\mathrm{t}^{2}}<\tanh (\mathrm{t})<\frac{\xi \mathrm{t}}{\xi+\mathrm{t}^{2}}
$$

with $\xi \approx 3.194528$. On integration, for $x \in(0,1)$, we have

$$
\int_{0}^{x} \frac{3 t}{3+t^{2}} d t<\int_{0}^{x} \tanh (t) d t<\int_{0}^{x} \frac{\xi t}{\xi+t^{2}} d t
$$

which implies that

$$
\frac{3}{2} \log \left(1+\frac{x^{2}}{3}\right)<\log (\cosh (x))<\frac{\xi}{2} \log \left(1+\frac{x^{2}}{\xi}\right) .
$$

The desired result follows by composing with the exponential function.

Acknowledgments: We would like to thank the referee for the thorough comments which have helped the presentation of the paper. 


\section{References}

[1] H. Alzer and M. K. Kwong, On Jordan's inequality, Period Math Hung, Volume 77, Number 2, pp. 191-200, 2018, doi: 10.1007/s10998-017-0230-z. [Online]. Available: https://doi.org/10.1007/s10998-017-0230z

[2] G. D. Anderson, M. K. Vamanamurthy and M. Vuorinen, Conformal Invarients, Inequalities and Quasiconformal maps, John Wiley and Sons, New York, 1997.

[3] Y. J. Bagul, On exponential bounds of hyperbolic cosine, Bulletin Of The International Mathematical Virtual Institute, Volume 8 , Number 2, pp. 365-367, 2018.

[4] Y. J. Bagul, New inequalities involving circular, inverse circular, hyperbolic, inverse hyperbolic and exponential functions, Advances in Inequalities and Applications, Volume 2018, Article ID 5, 8 pages, 2018, doi: 10.28919/aia/3556. [Online]. Available: https://doi.org/10.28919/aia/3556

[5] Y. J. Bagul, Inequalities involving circular, hyperbolic and exponential functions, J. Math. Inequal, Volume 11, Number 3, pp. 695-699, 2017, doi: 10.7153/jmi-2017-11-55. [Online]. Available: http://dx.doi.org/10.7153/jmi-2017-11-55

[6] Y. J. Bagul, On Simple Jordan type inequalities, Turkish J. Ineq., Volume 3, Number 1, pp. 1-6, 2019.

[7] Y. J. Bagul and C. Chesneau, Some sharp circular and hyperbolic bounds of $\exp \left(x^{2}\right)$ with Applications, preprint. hal-01915086. [Online]. Available: https://hal.archives-ouvertes.fr/hal01915086

[8] B. A. Bhayo, R. Klén and J. Sándor, New trigonometric and hyperbolic inequalities, Miskolc Mathematical Notes, Volume 18, Number 1, pp. 125-137, 2017, doi: 10.18514/MMN.2017.1560. [Online]. Available: https://doi.org/10.18514/MMN.2017.1560

[9] J. Cheeger, M. Gromov, M. Taylor, Finite propagation speed, kernel estimates for functions of the Laplace operator, and the geometry of complete Riemann manifolds, J. Differ. Geom., Number 17, 15-53, 1982.

[10] C. Chesneau, Some tight polynomial-exponential lower bounds for an exponential function, Jordan Journal of Mathematics and Statistics (JJMS), Volume 11, Number 3, pp. 273-294, 2018.

[11] C. Chesneau, On two simple and sharp lower bounds for $\exp \left(\mathrm{x}^{2}\right)$, preprint. hal-01593840. [Online]. Available: http://hal.archives-ouvertes.fr/hal-01593840 
[12] C. Chesneau, Y. J. Bagul, A note on some new bounds for trigonometric functions using infinite products, 2018, hal-01934571.

[13] C. Huygens, Oeuvres completes, Société Hollondaise des Sciences, Haga, 1888-1940.

[14] Y. Lv, G. Wang and Y. Chu, A note on Jordan type inequalities for hyperbolic functions , Appl. Math. Lett., Volume 25, Number 3, pp. 505-508, 2012, doi: 10.1016/j.aml.2011.09.046. [Online]. Available: https://doi.org/10.1016/j.aml.2011.09.046

[15] B. Malesevic, T. Lutovac and B. Banjac, One method for proving some classes of exponential analytic inequalities, preprint. arXiv:1811.00748v1. [Online]. Available: https://arxiv.org/abs/1811.00748

[16] E. Neuman and J. Sándor, On some inequalities involving trigonometric and hyperbolic functions with emphasis on the Cusa-Huygens, Wilker and Huygens inequalities, Math. Inequal. Appl., volume 13 Number 4, pp. 715723, 2010, doi: 10.7153/mia-13-50. [Online]. Available: http://dx.doi.org/10.7153/mia-13-50

[17] F. Qi, D.-W. Niu and B.-N. Guo, Refinements, generalizations and applications of Jordan's inequality and related problems, Journal of Inequalities and Applications, Volume 2009, Article ID 271923, 52 pages, 2009, doi: 10.1155/2009/271923. [Online]. Available: https://doi.org/10.1155/2009/271923

[18] J. Sándor, Sharp Cusa-Huygens and related inequalities, Notes on Number Theory and Discrete Mathematics, volume 19, Number 1, pp. 50-54, 2013.

[19] J. Sándor and R. Oláh-Gál, On Cusa-Huygens type trigonometric and hyperbolic inequalities, Acta Univ. Sapientiae, Mathematica, Volume 4, Number 2, pp. 145-153, 2012.

[20] Z.-H. Yang and Y.-M. Chu, Jordan type inequalities for hyperbolic functions and their applications, Journal of Function Spaces, Volume 2015, Article ID 370979, 4 pages, 2015, doi: 10.1155/2015/370979. [Online]. Available: http://dx.doi.org/10.1155/2015/370979

[21] L. Zhu, A source of inequalities for circular functions, Computers and Mathematics with Applications, Volume 58, Number 10, pp. 1998-2004, 2009, doi: 10.1016/j.camwa.2009.07.076. [Online]. Available: https://doi.org/10.1016/j.camwa.2009.07.076 